\title{
TOWARDS THE COMPUTATION OF VISCOUS FLOW RESISTANCE OF A LIQUID BRIDGE
}

\author{
C. $\mathrm{CHEN}^{1,2}$, P. DURU ${ }^{1}$, M. PRAT ${ }^{1,3}$, P. JOSEPH ${ }^{4,5}$ \& S. GEOFFROY ${ }^{2}$ \\ ${ }^{1}$ Université de Toulouse, INPT, UPS, IMFT, France. \\ ${ }^{2}$ Université de Toulouse, UPS, INSA, LMDC, France. \\ ${ }^{3} \mathrm{CNRS}$, IMFT, Toulouse, France. \\ ${ }^{4}$ CNRS, LAAS,France. \\ ${ }^{5}$ Université de Toulouse, LAAS, France.
}

\begin{abstract}
Flow within thick liquid films present owing to capillary effects in the pore space is of key importance in many multiphase flow applications in porous media, for example, drying or oil recovery processes. The viscous resistance to the flow is a key parameter for modelling fluid transport in such situations. It is well known for liquid films wetting the corners of tubes of polygonal cross-section. In this latter case, the liquid films shape is simple and can be readily obtained. The situation is much more involved when considering a realistic pore space, as in a packing of spherical particles, for example. In this case, X-ray tomography observations have shown that most of the liquid is confined around contact points between particles at intermediate liquid saturation. Nonetheless, a connectivity of all the liquid bridges throughout the particle packing can exist, allowing liquid transport across the porous medium. The ultimate goal of the present research is to provide the viscous flow resistance for such capillary liquid cluster of complex shape. As a first step in this direction, we present in this paper direct numerical simulation of the Stokes flow in liquid bridges obtained between two cylindrical pillars confined between two horizontal plates. The liquid bridge shape is obtained under conditions of hydrostatic equilibrium thanks to the Surface Evolver software. Then simulations of the viscous flow within the bridge are performed using Comsol Multiphysics ${ }^{\circledR}$ Creeping flow solver.

Keywords: capillary flow, liquid bridge, porous media, viscous resistance.
\end{abstract}

\section{INTRODUCTION}

Flows in thick liquid films trapped by capillary effects in the corners of the pore space can have a major impact on transport processes in porous media. For example, it has been shown that the overall drying time was orders of magnitude shorter in systems favoring the development of corner films, for example, [1-3]. However, the film model considered in these references was essentially the one developed for a straight tube of polygonal cross section. The pore space geometry in a porous medium is of course considerably more complex, and the thick films can have much more complex shapes as demonstrated for instance in [4] for random packing of particles. In this case, most of the liquid is confined around contact points between particles. Nonetheless, a connectivity of all the liquid bridges throughout the particle packing can exist, allowing flow and transport within the film network across the porous medium. Thus, this case can be envisioned as a system of interconnected liquid bridges. As a first step toward the computation of flow in such complex systems, we consider in the present paper a geometrical configuration somewhat simpler than the one expected in random packing of particles but significantly more complex than in straight tubes. As illustrated in Fig. 1, we consider the system formed by a series of liquid bridges

This paper is part of the Proceedings of the $8^{\text {th }}$ International Conference on Computational and Experimental Methods in Multiphase and Complex Flow (Multiphase Flow 2015)

www.witconferences.com
} 


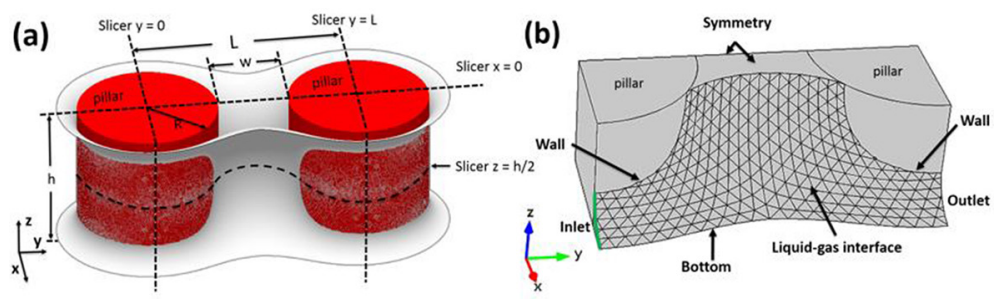

Figure 1: (a) Liquid bridge connecting two pillars confined between two horizontal plates (not shown in this sketch). The dimensionless liquid bridge volume is 0.46 (the reference volume is the volume of a pillar). The distance between the two horizontal plates is equal to the pillar diameter $d$ (the height of pillars is therefore equal to their diameter), whereas the gap $w$ between the two pillars is equal to the pillar radius so that the length of unit cell is $L=1.5 \mathrm{~d}$. (b) Computational domain (onefourth of unit cell is considered owing to symmetries).

connecting a straight array of cylindrical pillars confined between two horizontal plates. Figure 1 shows a representative unit cell of this chain of interconnected liquid bridges. In what follows, we consider the case where the distance $h$ between the two horizontal plates is equal to the pillar diameter $d$ (the height of pillars is therefore equal to their diameter), whereas the gap $w$ between the two pillars is equal to the pillar radius. Hence, the lattice spacing $L$, that is, the distance between the centers of two neighbor pillars, is equal to $1.5 d$.

The main objective is to compute the viscous flow resistance of the bridge as a function of its volume. As discussed in [1], this parameter is crucial to determine the spatial extent of liquid films (here, e.g., the maximum distance over which liquid bridges can be interconnected along the aforementioned straight array of pillars in the presence of a viscous flow). This information will be used in a future work devoted to the study of evaporation from arrays of cylinders connected by liquid bridges.

The shape of the liquid bridge is controlled only by capillary effects. This means that we are interested in situations where the viscous pressure drops over the unit cell due to the flow within the liquid bridge is negligible compared to the pressure jump across the liquid-gas interface due to capillarity (the capillary number is very small). As a result, the computational procedure can be decomposed into two main steps. The first step is to compute the shape of the liquid bridge for a given liquid volume under hydrostatic conditions. To this end, we use the energy minimizer software Surface Evolver (SE) [5], as explained in Section 2.1. Afterwards, the flow in liquid bridge is computed using the commercial code Comsol Multiphysics ${ }^{\circledR}$. This second step is described in Section 2.2.

\section{METHODS OF SOLUTION}

\subsection{Surface Evolver}

As mentioned in the introduction, the first step in the present work consists in computing the shape of the liquid bridge. To this end, we use the SE software. Starting from an initial 'guess' shape for the static liquid bridge, SE progressively converges towards the shape minimizing the surface energy by a gradient descent method. During the evolution procedure, the surface can be progressively refined by the user, by increasing the number of facets that shape it.

Gravitational effects are neglected. The contact angle between the liquid and the top and bottom plates can be adjusted freely. Note that the liquid is initially confined into a parallel 
lipipedic volume enclosing the two pillars. For small enough liquid volumes, it is found after surface energy minimization that the liquid does not wet some part of the pillars surface. Note that a contact angle of $0^{\circ}$ is imposed on the pillar surface by SE. Imposing the same contact angle than the one imposed on the top and bottom plates would require to start with another initial shape. This will be the subject of a future work. The volume of liquid confined between the two cylindrical pillars is another control parameter. In the present study, the total volume of the liquid bridge, made dimensionless by a single pillar volume, was taken to vary between 0.24 and 0.99 . For such volumes, there is always a liquid bridge between the two pillars, that is, the liquid is not split into disconnected liquid clusters around each pillar.

A typical final shape is illustrated in Fig. 1a. The surface of the pillars is displayed in red colour. To ease the capillary surface visualization, the top and bottom plates are not displayed. The surface mesh display has also been turned off. The computational domain to be used in Comsol (see Section 2.2) is shown in Fig. 1b. It consists, for symmetry reasons, in a quarter of the liquid bridge unit cell, that is confined between the plane $y=0$ and the plane $y=L$.

As can be seen from Fig. 1a, we actually consider an isolated system formed by two pillars (as opposed to a spatially periodic system of pillars). It is surmised that the computed shape is very close to the one corresponding to a spatially periodic system of pillars in the $y$ direction. However, this remains to be confirmed.

\subsection{Viscous flow simulation}

The wetting fluid is supposed isothermal, incompressible and Newtonian with constant dynamic viscosity $\mu$ and constant density $\rho$. Due to small dimensions, the Reynolds number is far less than 1, so that inertial terms can be neglected and gravity is not considered. From these assumptions, the equations of motion reduce to Stokes equations. Continuity and momentum equations can thus be expressed as

$$
\begin{gathered}
\nabla . \mathrm{u}=0 \\
\mu \nabla^{2} \mathrm{u}-\nabla P_{l}=0
\end{gathered}
$$

where $\mathbf{u}$ is the liquid velocity and $P_{1}$ the pressure in the liquid.

Equations (1) and (2) are solved numerically using the finite element based commercial software COMSOL Multiphysics ${ }^{\circledR}$ in the liquid domain depicted in Fig. 1b. Free tetrahedral elements are used for meshing the liquid domain. A no-slip boundary condition is implemented on the liquid-pillar and liquid-bottom plate interfaces. No-viscous stress pressure boundaries are imposed at the computational inlet and outlet boundaries. Similarly, as in a previous work for a simpler geometry, that is [6], two different boundary conditions are used and compared at the liquid-gas interface, namely the no-stress boundary condition or the no-slip boundary condition so as to simulate a perfectly clean interface or a rigidified interface due to surfactant for instance. The computational mesh is refined until obtaining results independent of meshing.

\section{RESULTS AND DISCUSSION}

\subsection{Validation of the numerical procedure}

Before consideration of liquid bridges, the numerical procedure combining SE and Comsol computations was tested for the much simpler geometrical configuration considered in [6], 
namely the flow in a liquid wedge trapped by capillarity in a right angle straight corner. This case is illustrated in Fig. 2a. As in [6] the viscous resistance within the liquid wedge is expressed by the dimensionless flow resistance $\beta$ defined as

$$
\beta=\frac{R^{2}}{\mu\left\langle u_{y}\right\rangle}\left(-\frac{d P_{l}}{d y}\right)
$$

where $\left\langle u_{y}\right\rangle$ is the average velocity in the wedge in the longitudinal direction, calculated by dividing the flow rate in the wedge by the liquid corner area, $R$ is the interface curvature radius in the $x-z$ plane in Fig. $2 \mathrm{a}$ (the interface is flat in the $y$ direction), $\frac{d P_{l}}{d y}$ is the pressure
gradient driving the flow in the liquid wedge.

The meniscus surface is first computed using SE in a right angle corner as illustrated in Fig. 2 a varying the contact angle implemented on the $y-z$ and $x-y$ planes in SE. The cross-section profile of meniscus is then imported into Comsol and then the liquid wedge is rebuilt with an extrude tool available in Comsol to construct a mesh more adapted to the viscous flow computation. The vertical and bottom wall are set as no-slip boundary, the two cross-sections along $y$ axis are set as inlet and outlet. A pressure difference $\Delta P_{1}$ is imposed between the inlet and the outlet. The no viscous stress or the no-slip boundary condition is imposed at the liquid-gas interface.

After computation of the flow using Comsol, the viscous resistance is computed using eqn (3). This was performed by varying the contact angle. The comparison between our computations and the results of [6] is shown in Fig. 2b. The red lines in Fig. 2b correspond to the flow resistance results obtained using our procedure, whereas the blue ones are the ones from Ref. [6]. Black and blue lines correspond to no-slip boundary, and red and green lines correspond to no-stress boundary conditions at liquid-gas interface, respectively. As can be seen, there is an almost perfect agreement between our results and the ones of [6].

\subsection{Capillary pressure versus liquid bridge volume}

The SE computation was first made by varying the volume of the liquid bridge present in the unit cell. As expected and shown in Fig. 3b, increasing the volume reduces the pressure jump
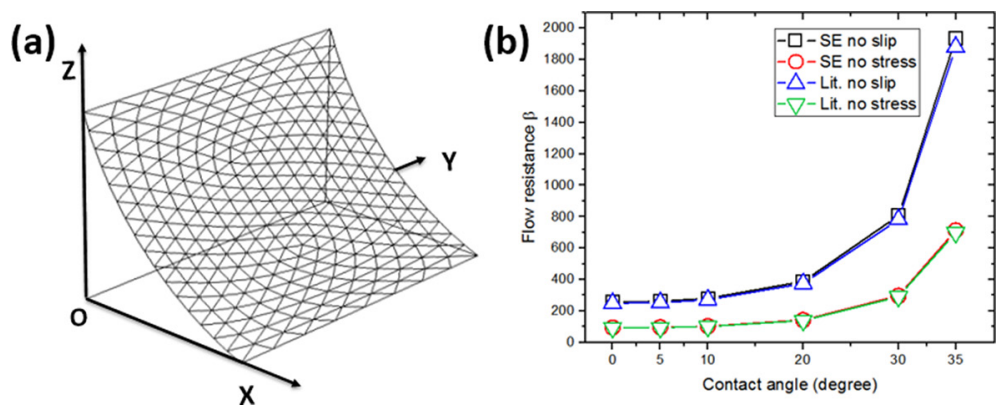

Figure 2: (a) Example of liquid-gas interface shape in a right angle straight corner computed using Surface Evolver. (b) Variation in flow resistance with contact angle for the straight corner flow configuration. Comparison between the present computations (referred to as SE) and the results of [6] (referred to as 'Lit.'). 

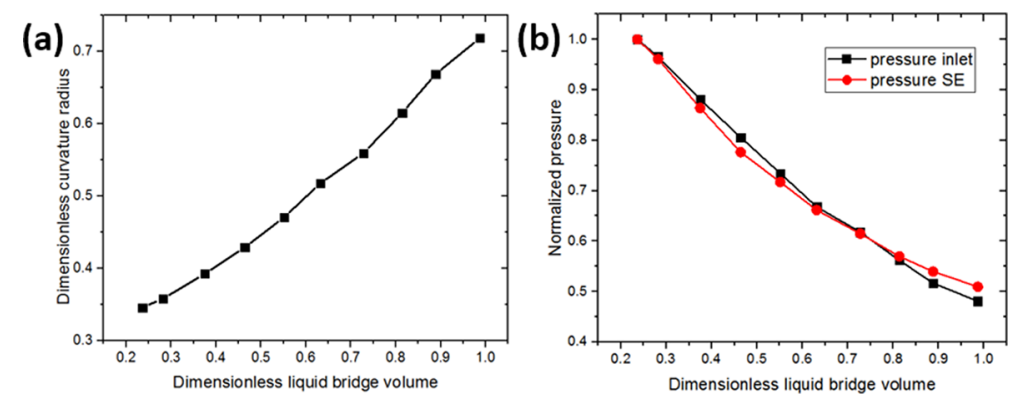

Figure 3: Curvature radius $R_{\text {in }}$ at unit cell inlet and capillary pressure as a function of liquid bridge volume in unit cell (made dimensionless using the volume of a pillar): (a) curvature radius $R_{\text {in }}$ in the $x-z$ plane at the unit cell inlet (this corresponds to the green line in Fig. 1b) made dimensionless using the pillar radius $d / 2$ as characteristic length. (b) Capillary pressure $P_{\mathrm{c}}$ obtained with SE (red dot line) together with the pressure jump (black dot line) computed as $P_{\text {cinlet }}=\frac{\gamma \cos \theta}{R_{\text {in }}}$ and made dimensionless using $P_{c r e f}=\frac{\gamma \cos \theta}{(d / 2)}$ as the reference pressure (these results are for $\theta=10^{\circ}$ ).

between the liquid and the gas phase due to capillarity. The pressure jump is the capillary pressure $P_{\mathrm{c}}$ defined by $P_{\mathrm{c}}=P_{\mathrm{g}}-P_{\mathrm{l}}$, where $P_{\mathrm{g}}$ and $P_{1}$ are the pressures in the liquid and the gas phases, respectively. The local shape of the liquid-gas interface is also characterized by the curvature radius of the liquid-gas interface in the $x-z$ plane at the unit cell inlet (this corresponds to the green line in Fig. 1b. The results are shown in Fig. 3a, where the volume of the unit cell liquid bridge and the considered curvature radius are made dimensionless using the volume of a pillar and the pillar radius, respectively. As can be seen from Fig. $3 b$, the capillary pressure can be actually computed simply from the determination of $R_{\text {in }}$.

\subsection{Pressure distribution in liquid bridge}

The pressure distribution induced by creeping viscous flow within the bridge is illustrated in Fig. $4 \mathrm{a}$ for the case $q=10^{\circ}$ and a dimensionless liquid bridge volume in unit cell of 0.46 . The pressure field is further illustrated in Fig. 4b, which shows the pressure (blue line) and the pressure gradient along the bottom triple line of Fig. 4a. As illustrated in Fig. 4a, one can distinguish two main regions in the liquid bridge: the central region between the two pillars where the liquid bridge cross-section areas in the $x-z$ plane are relatively large and the constriction regions at the inlet and outlet of the unit cell where the liquid bridge is in contact with the pillars. As can be seen, the pressure gradient is concentrated in the inlet and outlet regions, that is, the constriction regions of the bridge. The pressure variation is significantly weaker in the central region of the bridge between the pillars. The flow resistance in this example is thus mostly concentrated in the inlet and outlet regions of the bridge.

\subsection{Liquid bridge viscous resistance}

Similarly as for the flow in a straight corner (see Section 3.1), the viscous flow resistance $\beta$ of the bridge is defined by 
(a)

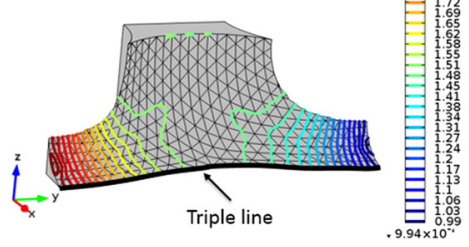

(b)

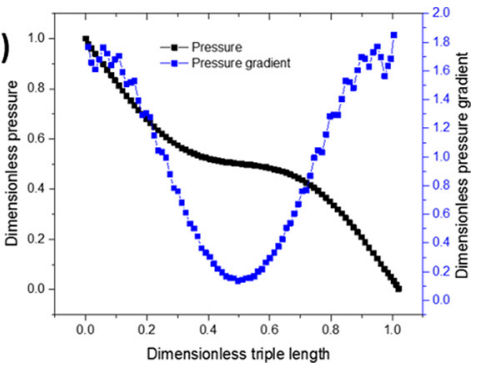

Figure 4: (a) Example of pressure distributions (isobars on the liquid-gas interface, where b.c. is set as no-stress boundary condition) in a liquid bridge of dimensionless volume 0.46. (b) Distribution of dimensionless pressure (defined as $P_{l}^{*}=\frac{P_{l}-P_{\text {loutlet }}}{\Delta P_{l}}$ ) and distribution of pressure gradient in the liquid along the triple line indicated in Fig. 3a. The distance along the triple line is made dimensionless using the unit cell length $L$ (see Fig. 1), the dimensionless pressure gradient along the triple line is defined as $\frac{\partial P_{l}}{\partial s} /\left(\Delta P_{l} / L\right)$, here $s$ is a curvilinear coordinate along the triple line.

$$
\beta=\frac{R_{\text {in }}^{2}}{\mu\left\langle u_{y}\right\rangle}\left(\frac{\Delta P_{l}}{L}\right)
$$

where $R_{\text {in }}$ is the curvature radius at unit cell inlet discussed previously (see Section 3.2). Figure 5 shows the variation in the viscous flow resistance with the volume of liquid bridge computed for $\theta=10^{\circ}$. The three insets show the liquid bridge shape observed from above (top view) corresponding to dimensionless volume $0.24,0.46$ and 0.99 , respectively. As can be seen, the shape of liquid bridge significantly changes when the liquid bridge volume is varied. The inlet curvature radius $R_{\text {in }}$ gradually increases with increasing liquid bridge volume, which is also illustrated in Fig. 3a. We also observed that in bridge central region (between pillars) the curvature radius increases with increasing liquid bridge volume, implying that the pressure in the liquid bridge decreases, in accordance with the results shown in Fig. 3b.

As can be seen from Fig. 5, the dimensionless flow resistance of liquid bridge as defined by eqn (4) is of the same order of magnitude as the reference dimensionless resistance corresponding to a flow in a right angle straight corner. Table 1 shows a detailed comparison between our computations and the case of a right angle strait corner for the special case corresponding to the bridge depicted in Fig. 4 (dimensionless liquid bridge volume $=0.46$ ). As can be seen, the flow resistances of the liquid bridge are less than the ones for the right angle straight corner for this liquid bridge volume. This can be qualitatively understood from Fig. $4 \mathrm{~b}$, which shows that the pressure drop is significantly less in the central region of the bridge. Hence, considering a reduced length in eqn (4), that is, a length lower than $L$ corresponding to the length over which the pressure drop is significant, would lead to increased $\beta$. However, it is not obvious to define such a reduced length only from bridge geometry.

Furthermore, as shown in Fig. 5, the bridge flow resistance varies with liquid bridge volume and can be greater or smaller than for the right angle straight corner resistance depending on the liquid bridge volume. The tendency shown in Fig. 5 is consistent with the bridge shape variation with its volume depicted in Fig. 5. The bridge is more tortuous for the 


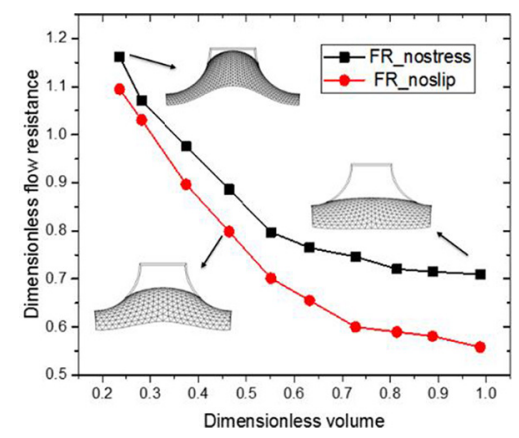

Figure 5: Liquid bridge flow resistance $\beta / \beta_{\text {ref }}$ and liquid bridge shape variation with increasing liquid bridge volume in unit cell. Solid line with square symbols corresponds to results obtained with the no viscous stress boundary condition at the liquid-gas interface, solid line with circles is for the no-slip boundary condition imposed at the liquid-gas interface. The liquid bridge flow resistance is divided by the flow resistance $\beta_{\text {ref }}$ for a right angle corner flow documented in [6] $\left(\beta_{\text {ref }}=93.93\right.$ for the no-stress boundary condition and $\beta_{\text {ref }}=253.2$ for the no-slip boundary condition and $\theta=10^{\circ}$ ).

Table 1: Comparison of dimensionless flow resistance $\beta$ between liquid bridge model and right angle straight corner model for two values of contact angle for a liquid bridge of dimensionless volume 0.46 .

\begin{tabular}{|c|c|c|c|c|}
\hline \multirow{3}{*}{ Contact angle } & \multicolumn{4}{|c|}{ Flow resistance } \\
\hline & \multicolumn{2}{|c|}{ Interface no stress b.c. } & \multicolumn{2}{|c|}{ Interface no slip b.c. } \\
\hline & Liquid bridge & Straight corner & Liquid bridge & Straight corner \\
\hline 0 & 83.22 & 93.93 & 201.47 & 248.8 \\
\hline 10 & 83.26 & 93.93 & 202.13 & 253.2 \\
\hline
\end{tabular}

smaller volumes, which is consistent with the increase of the flow resistance with decreasing bridge volume. For the larger volumes tested, the flow resistance is less than for a right angle straight corner. This is consistent with the fact that the shape is not tortuous anymore (see the shape on the right-hand side in Fig. 5) and less vertical wall is wetted comparatively with a straight corner of same length.

\section{CONCLUSION}

We have described a method for computing the viscous flow in a capillary bridge attached by capillarity to two inline pillars confined between two horizontal plates. The method combined two software. The first one is used to compute the bridge shape under capillary equilibrium conditions, whereas the second one is used to compute the viscous flow within the liquid bridge. This method is of course restricted to situations where the pressure jump across the liquid-gas interface due to capillarity is much larger than the viscous pressure drop within the bridge. The study shows that the dimensionless flow resistance of the bridge can 
be roughly estimated from the well-documented flow resistance in a right angle straight corner, using the curvature radius of the liquid-gas interface in the cross-section of smallest area along the bridge.

Further investigations are needed to try to define a simple but more accurate approach to this resistance, which actually depends on the liquid bridge volume as well as other parameters such as the pillar spacing or the pillar aspect ratio. The latter were not varied in the present studies but can be shown to have an impact on the flow resistance using the computational approach presented in the present paper.

The presented results are currently exploited to analyze evaporation experiments in microfluidic devices. They also open up the route toward the computation of the flow in the more complex chains of liquid bridges forming in granular porous media.

\section{REFERENCES}

[1] Chauvet, F., Duru, P., Geoffroy, S. \& Prat, M., Three periods of drying of a single square capillary tube. Phys. Rev. Lett. 103, pp. 124502, 2009. doi: http://dx.doi.org/10.1103/ PhysRevLett.103.124502

[2] Prat, M., On the influence of pore shape, contact angle and film flows on drying of capillary porous media. Int. J. Heat Mass Transfer 50, pp. 1455-1468, 2007. doi: http:// dx.doi.org/10.1016/j.ijheatmasstransfer.2006.09.001

[3] Yiotis, A.G., Salin, D., Tajer, E.S. \& Yortsos, Y.C., Drying in porous media with gravity stabilized fronts: experimental results. Phys. Rev. E 86, pp. 026310, 2012. doi: http:// dx.doi.org/10.1103/PhysRevE.86.026310

[4] Scheel, M., Seemann, R., Brinkmann, M., Michiel, M.D., Sheppard, A. \& Herminghaus, S., Liquid distribution and cohesion in wet granular assemblies beyond the capillary bridge regime. J. Phys. Condensed Matter 20, pp. 494236, 2008. doi: http://dx.doi.org/10.1088/0953-8984/20/49/494236

[5] Brakke, K., The Surface Evolver. Exp. Math., 1(2), pp. 141-165, 1992. doi: http:// dx.doi.org/10.1080/10586458.1992.10504253

[6] Ransohoff, T.C. \& Radke, C.J., Laminar flow of a wetting liquid along the corners of a predominantly gas-occupied noncircular pore. J. Colloid Interface Sci. 121(2), pp. 392-401, 1988. doi: http://dx.doi.org/10.1016/0021-9797(88)90442-0 